MS17-P03

\section{Multi-scale characterization of bone mineral composition and structural organization}

Pedro Alvarez ${ }^{1}$, Nazaret Domínguez-Gasca ${ }^{2}$, Cristina Benavides ${ }^{2}$, Estefanía Sánchez-Rodríguez ${ }^{2}$, Alejandro Rodríguez-Navarro ${ }^{2}$

1. Deparmento de Geología; Universidad de Oviedo, Oviedo, Spain

2. Departamento de Mineralogia y Petrologia, Universidad de Granada, Granada, Spain

email: pedroalvarez@uniovi.es

Bone is a complex biocomposite material constituted by carbonated apatite nanocrystals deposited in an oriented fashion and integrated within an organic matrix, mostly collagen fibrils. To define how bone compositional and structural characteristics in different bone types (i.e. cortical and trabecular bone) affect relevant bone properties (i.e., mechanical properties), we have applied complementary analytical techniques such as electron microscopy, computed tomography, infrared spectroscopy and $\mathrm{X}$ ray diffraction. These techniques provide detailed information about the mineral and organic components of bone tissue and allow the determination of its porosity, degree of mineralization, carbonate content, mineral crystallinity and collagen cross-linking. Bone compositional parameters determined by infrared spectroscopy (e.g. degree of mineralization (PO4/Amide I), the total carbonate (CO4_1415/Amide I) are important parameters to define bone material quality and are useful to detect possible metabolic alterations associated to specific bone pathological conditions (osteoporosis, osteomalicia). BSE-SEM images of cross-section at mid-diaphysis showed notable differences in the organization of different bone types (cortical and trabecular bone) and bone structures at different scales. Analyses by means 2D X-ray diffraction showed differences between types of bone related to mineral organization and its crystallinity. The degree of alignment of the c-axis of apatite crystals can be determined from the intensity profile along Debye-Scherrer ring associated with the 002 reflection. The preferential orientation of the apatite crystals is due to an aligned arrangement of collagen fibers in cortical organization while the crystals showed randomly orientation at trabecular level. The information obtained allowed us to determine the influence of chemical composition and microstructural organization on cortical and trabecular regions in the overall bone material properties.

Keywords: bone, organization, microstructure
MS17-P04

\section{Structural evolution of hydrated calcium oxalates}

Alina Izatulina ${ }^{1}$, Vladislav Gurzhiy ${ }^{1}$, Maria Krzhizhanovskaya ${ }^{1}$, Mariya Kuz'mina ${ }^{1}$, Matteo Leoni², Olga Frank-Kamenetskaya ${ }^{1}$

1. Department of Crystallography, St. Petersburg State University, St. Petersburg, Russia

2. University of Trento, Trento, Italy

email: alina.izatulina@mail.ru

Calcium oxalates are represented in nature by three hydrated forms: whewellite $\left(\mathrm{CaC}_{2} \mathrm{O}_{4} \cdot \mathrm{H}_{2} \mathrm{O} ; \mathrm{COM}\right)$, weddellite $\left(\mathrm{CaC}_{2} \mathrm{O}_{4} \cdot(2+x) \mathrm{H}_{2} \mathrm{O}\right.$; COD) and caoxite $\left(\mathrm{CaC}_{2} \mathrm{O}_{4} \cdot 3 \mathrm{H}_{2} \mathrm{O}\right.$; COT). Calcium oxalates are also found among the pathogenic mineral precipitates in human bone marrow, myocardium, joints, lungs, liver, thyroid gland, intestinal mucosa, eyes, and urinary system. Oxalates span therefore several fields (medicine, biology, mineralogy, materials science, etc.), which is reflected in a large number of publications. Nevertheless, many questions remain unresolved. For instance, the role of water in the formation of calcium oxalate crystal structures as well as the mechanisms of phase transition is still unclear.

Thermal stability, structural evolution pathways and phase transition mechanisms of the calcium oxalates COM, COD and COT have been analyzed using single crystal and powder X-ray diffraction techniques at non ambient conditions. The reduction of $\mathrm{H}_{2} \mathrm{O}$ content in the structures increases dimensionality from dimers and chains to the layered structural units and from rarefied to denser sheets within the compounds whose structures are based on the 2D units. While studying the phase transitions pathways within the calcium oxalate family, two crystalline compounds have been structurally characterized for the first time $\left(\alpha-\mathrm{CaC}_{2} \mathrm{O}_{4}\right.$ and $\mathrm{CaC}_{2} \mathrm{O}_{4} \cdot \mathrm{H}_{2} \mathrm{O}$ ), among which the novel COM modification has been obtained for the first time as well. The highest thermal expansion of these compounds is observed along the direction of the hydrogen bonds, whereas the lowest expansion and even contraction of the structures occur due to the displacement of neighbor layered complexes towards each other and to an orthogonalization of the monoclinic angles. Within the calcium oxalate family, whewellite should be regarded as the most stable crystalline phase at ambient conditions. Weddellite and caoxite transform to whewellite during dehydration-driven phase transition promoted by time and/or heating. Finally, we'd like to emphasize the particular importance of the structural and chemical evolution studies of calcium oxalates, as their phase transitions occur at temperatures typical of the human body and can therefore have a significant effect on health.

This work was supported by President of Russian Federation grant for leading scientific schools (no. NSh3079.2018.5), Russian Foundation for Basic Research (no. 16-05-00986) and by the People Program (Marie Curie Actions) of the European Union's Seventh Framework Program FP7/2007-2013/ under REA grant agreement IRSESGA-2013-610547-TAMER. The XRD measurements have been performed at the X-ray Diffraction Centre of St. Petersburg State University.

Keywords: calcium oxalate, crystal strusture, phase transition 